\title{
Piercing Numbers for Balanced and Unbalanced Families
}

\author{
Luis Montejano • Pablo Soberón
}

Received: 13 December 2009 / Revised: 25 February 2010 / Accepted: 25 August 2010 /

Published online: 9 September 2010

(C) Springer Science+Business Media, LLC 2010

\begin{abstract}
Given a finite family $\mathcal{F}$ of convex sets in $\mathbb{R}^{d}$, we say that $\mathcal{F}$ has the $(p, q)_{r}$ property if for any $p$ convex sets in $\mathcal{F}$ there are at least $r q$-tuples that have nonempty intersection. The piercing number of $\mathcal{F}$ is the minimum number of points we need to intersect all the sets in $\mathcal{F}$. In this paper we will find some bounds for the piercing number of families of convex sets with $(p, q)_{r}$ properties.
\end{abstract}

\section{Introduction}

Given a finite family $\mathcal{F}$ of convex sets in $\mathbb{R}^{d}$, the piercing number, $\pi(\mathcal{F})$, of $\mathcal{F}$ is the minimum number $m$ such that there is a set of $m$ points that intersects every nonempty set of $\mathcal{F}$.

Helly's theorem characterizes families of nonempty convex sets with piercing number one. So, it is natural to ask if by weakening the Helly's condition we still obtain an upper bound for the piercing number of the family. One way to weaken this condition is to use the $(p, q)$ property, that is, to say that for every $p$ convex sets of the family there are $q$ which are intersecting. It was conjectured by Hadwiger and Debrunner in 1957 [3] that for every $p, q, d$, such that $p \geq q \geq d+1$, there is a $c=c(p, q, d)$ such that every finite family $\mathcal{F}$ of convex sets in $\mathbb{R}^{d}$ with the $(p, q)$ property has $\pi(\mathcal{F}) \leq c$. This result was proved in 1992 by Alon and Kleitman [1]. The bounds given by this proof are by no means optimal.

Finding good bounds for the piercing number of families with the $(p, q)$ property has proven to be extremely difficult. The first nontrivial case is the $(4,3)$ property in $\mathbb{R}^{2}$. It is still open. The conjecture is that any finite family of plane convex sets with

Supported by CONACYT, 41340.

L. Montejano $(\varangle) \cdot$ P. Soberón

Instituto de Matemáticas, Universidad Nacional Autónoma de México, México D.F., Mexico

e-mail: luis@math.unam.mx 
the $(4,3)$ property can be pierced with 3 points. The best bound that has been proven is 13 points [4].

In dimension 1 , there is an optimal bound. If $\mathcal{F}$ is a finite family of at least $p$ intervals with the $(p, q)$ property, $p \geq q \geq 2$, then $\pi(\mathcal{F}) \leq p-q+1$. We include the proof because we will follow its spirit in the proofs of our main theorems. We use induction on $p-q$. If $p=q$, we have Helly's condition on the line. If $\mathcal{F}$ has the $(p, q)$ property with $p>q$, we can consider $I$ the interval with its leftmost point $p_{0}$ being rightmost to all the others. If we take $B$ to be the set of intervals that do not intersect $I$, then we have two cases. If there are at least $p-1$ intervals in $B$, then every $p-1$ of them and $I$ must have $q$ which are intersecting. Those $q$ cannot contain $I$, so $B$ has the $(p-1, q)$ property and thus can be pierced with $p-q$ points. Adding $p_{0}$, we have the result. If there are less that $p-1$ sets in $B$, then we can take all the intervals in $B, I$ and some of the other intervals to have $p$ convex sets. These must have an intersecting $q$-tuple. If this $q$-tuple has $I$, then it cannot have any interval in $B$, thus $B$ has at most $p-q$ convex sets. Placing one point in every set of $B$ and $p_{0}$ gives the result. If this $q$-tuple does not have $I$, then it is missing at most $p-q-1$ sets of $B$. We can place one point for every one of those intervals, the point of intersection of the $q$-tuple and $p_{0}$.

This result is optimal since we can take a family $\mathcal{F}$ of $p-q$ pairwise disjoint intervals and then a set of intersecting intervals. This family has the $(p, q)$ property but cannot be pierced with less than $p-q+1$ points.

Definition 1 Let $p, q, r$ be positive integers, $p>q$. A finite family $\mathcal{F}$ of sets has the $(p, q)_{r}$ property if for every $p$ sets of $\mathcal{F}$, at least $r$ of its $q$-tuples are intersecting. If $0<\alpha<1$ is a real number, a finite family $\mathcal{F}$ of sets has the $(p, q)^{\alpha}$ property if for every $p$ sets of $\mathcal{F}$, the proportion of its intersecting $q$-tuples is greater than $\alpha$.

The purpose of this paper is to give bounds for the piercing number, $\pi(\mathcal{F})$, of a finite family $\mathcal{F}$ of convex sets in $\mathbb{R}^{d}$ with the $(p, q)_{r}$ and the $(p, q)^{\alpha}$ properties.

The following geometric lemma will be useful in the proofs of our main theorems.

Lemma 1 Let $\left\{A_{0}, \ldots, A_{\lambda}\right\}$ be a nonintersecting family of compact convex sets in $\mathbb{R}^{d}, 1 \leq \lambda \leq d$. Then there is an affine linear subspace $\Pi$ of $R^{d}$ of dimension $d-\lambda$ with the following property: if $X$ is a convex set and for every $i=0, \ldots, \lambda$, there is a point of $X$ in $\bigcap_{j \neq i} A_{j}$, then $X \cap \Pi \neq \phi$.

Proof We may assume without loss of generality that for every $i=1, \ldots, \lambda$, $\bigcap_{j \neq i} A_{j} \neq \phi$; otherwise, the conclusion follows immediately. We shall start proving by induction that $\bigcup_{0}^{\lambda} A_{j}$ has the homology of $\mathbb{S}^{\lambda-1}$, the sphere of dimension $\lambda-1$. If $\lambda=1$, then $\left\{A_{0}, A_{1}\right\}$ are two disjoint convex sets and, hence, $A_{0} \cup A_{1}$ has the homotopy type of $\mathbb{S}^{0}$. Suppose our claim is true for $\lambda-1$; we shall prove it for $\lambda$. The space $A=\bigcup_{1}^{\lambda} A_{j}$ is starshaped because every $A_{j}$ is convex and $\bigcap_{1}^{\lambda} A_{j} \neq \phi$. So, $\bigcup_{0}^{\lambda} A_{j}$ is the union of two contractible spaces, $A$ and $A_{0}$, whose intersection is $\bigcup_{1}^{\lambda} B_{j}$, where $B_{j}=A_{0} \cap A_{j}$. Note that for every $i=1, \ldots, \lambda, \bigcap_{j \neq i} B_{j} \neq \phi$ but $\cap_{1}^{\lambda} B_{j}=\phi$. Hence, by induction $A_{0} \cap A$ has the homology of $\mathbb{S}^{\lambda-2}$. Thus we have that $A_{0} \cup A$ is the union of two contractible spaces whose intersection has the homol- 
ogy of $\mathbb{S}^{\lambda-2}$. The same happens when we take $\mathbb{S}^{\lambda-1}$ as the union of two hemispheres. Then, using the Mayer-Vietoris exact sequence, $A \cup A_{0}$ has the homology of $\mathbb{S}^{\lambda-1}$.

We are ready to prove our lemma when $\lambda=d$. By the above, $\bigcup_{0}^{d} A_{j} \subset \mathbb{R}^{d}$ has the homology of $\mathbb{S}^{d-1}$ and hence $\mathbb{R}^{d}-\bigcup_{0}^{d} A_{j}$ has exactly two components, one of them bounded. Let $\Pi=\left\{v_{0}\right\}$, where $v_{0}$ is any point of the bounded component of $\mathbb{R}^{d}-\bigcup_{0}^{d} A_{j}$. For every $i=0, \ldots, d$, take $a_{i} \in \bigcap_{j \neq i} A_{j} \cap X$. Note that $\left\{a_{0}, \ldots, a_{d}\right\} \subset$ $\mathbb{R}^{d}$ is in general position, otherwise by Radon's theorem, $\bigcap_{0}^{\lambda} A_{j} \neq \phi$.

Let $\Delta$ be the convex hull of $\left\{a_{0}, \ldots, a_{d}\right\}$ and note that bd $\Delta \subset \bigcup_{0}^{d} A_{j}$. For every $i=0, \ldots, \lambda$, let $C_{i}=A_{i} \cap \Delta$. Hence, for every $i=1, \ldots, \lambda, \bigcap_{j \neq i} C_{j} \neq \phi$ but $\bigcap_{0}^{\lambda} C_{j}=\phi$. By the first paragraph of this proof, $\bigcup_{0}^{d} C_{j} \subset \mathbb{R}^{d}$ has the homology of $\mathbb{S}^{d-1}$, and hence $\mathbb{R}^{d}-\bigcup_{0}^{d} C_{j}=\Delta-\bigcup_{0}^{d} A_{j}$ has the homology of $\mathbb{S}^{d-1}$ and therefore has exactly two components. Furthermore, the bounded component of $\Delta-\bigcup_{0}^{d} A_{j}$ is contained in the interior of the simplex $\Delta$. So, the bounded component of $\mathbb{R}^{d}-\bigcup_{0}^{d} A_{j}$ is contained in the interior of the simplex $\Delta$, and hence $v_{0} \in X$, as we wished.

Let now $\lambda<d$. By Lemma 3.1 of [2], there is a $\lambda$-dimensional linear subspace $\Gamma$ of $\mathbb{R}^{d}$, with the property that if $\pi: \mathbb{R}^{d} \rightarrow \Gamma$ is the orthogonal projection, then $\bigcap_{0}^{\lambda} \pi\left(A_{j}\right)=\phi$. If $X \subset \mathbb{R}^{d}$ is a convex set and for every $i=0, \ldots, \lambda$ there is a point of $X$ in $\bigcap_{j \neq i} A_{j}$, then by the above paragraph, since for every $i=0, \ldots, \lambda$ there is a point of $\pi(X)$ in $\bigcap_{j \neq i} \pi\left(A_{j}\right)$, there is $v_{0} \in \Gamma$ such that $v_{0} \in \pi(X)$. Then $\Pi=$ $\pi^{-1}\left(v_{0}\right)$ is such that $\Pi \cap X \neq \phi$.

\section{Balanced Families}

Some good bounds for the piercing number of families with the $(p, q)_{r}$ property can be obtained if we impose an estimation on how well distributed the intersecting $q$ tuples are. Let $\mathcal{F}$ be a collection of sets. An $s$-balanced covering of $\mathcal{F}$ is a collection $\left\{\mathcal{F}_{1}, \ldots, \mathcal{F}_{r}\right\}$ of subsets of $\mathcal{F}$ such that $\bigcup_{1}^{r} \mathcal{F}_{i}=\mathcal{F}$ and every set of $\mathcal{F}$ is in at least $s$ of the $\mathcal{F}_{i}$ 's.

Lemma 2 Let $\left\{\mathcal{F}_{1}, \ldots, \mathcal{F}_{r}\right\}$ be an $(r-1)$-balanced covering of $\mathcal{F}$. Then every subfamily $G$ of $\mathcal{F}$ of size $r-1$ is contained in $\mathcal{F}_{i}$, for some $i=1, \ldots, r$.

Proof Every one of the $r-1$ sets of $G$ is in at least $r-1$ of the $\mathcal{F}_{i}$ 's, thus there is at most one of the $\mathcal{F}_{i}$ 's not containing it. So, we have at most $r-1$ of the $\mathcal{F}_{i}$ 's missing at least one of the original sets of $\mathcal{F}$. Thus, there is at least one of the $\mathcal{F}_{i}$ 's containing all the sets of $G$.

Definition 2 The family $\mathcal{F}$ has the $s$-balanced $(p, q)_{r}$ property if, given any subfamily $\mathcal{F}^{\prime} \subset \mathcal{F}$ of size $p$, we can find $r$ intersecting $q$-tuples of $\mathcal{F}^{\prime}$ and furthermore the $r$ intersecting $q$-tuples are an $s$-balanced covering of $\mathcal{F}^{\prime}$.

Sometimes, when $\mathcal{F}$ is a finite family of convex sets in $\mathbb{R}^{d}$, it is convenient to think of the $s$-balanced $(p, q)_{r}$ property as follows: the family $\mathcal{F}$ has the $s$-balanced $(p, q)_{r}$ property if and only if, given any subfamily $\mathcal{F}^{\prime}=\left\{S_{1}, \ldots, S_{p}\right\} \subset \mathcal{F}$, there are $r$ elements $\left\{\rho_{1}, \ldots, \rho_{r}\right\} \subset \bigcup_{1}^{p} S_{i}$, such that every $\rho_{i}, i=1, \ldots, r$, is in the in- 
tersection of $q$ sets of $\mathcal{F}^{\prime}$ and every $S_{i}, i=1, \ldots, p$, contains at least $s$ elements of $\left\{\rho_{1}, \ldots, \rho_{r}\right\}$.

By Lemma 2, we have that if a family of convex sets has the $(r-1)$-balanced $(p, q)_{r}$ property, then all $r-1$ sets of the family are intersecting.

Lemma 3 Let, $p, q, d$ be positive integers such that $p>q, q d>p(d-1)+1$ and let $\mathcal{F}$ be a finite family of at least $p$ convex sets in $\mathbb{R}^{d}$. If there is a subset $A \subset \mathcal{F}$ of $d$ nonintersecting convex sets and $\mathcal{F}$ has the $(d-1)$-balanced $(p, q)_{d}$ property, then

$$
\pi(\mathcal{F} \backslash A) \leq d(p-q-1)+1 .
$$

Proof Let $K_{1}, K_{2}, \ldots, K_{d}$ be the nonintersecting $d$-tuple. We know by Lemma 1 that there is a line $l$ such that every convex set $K$, that has nonempty intersection with every $(d-1)$-tuple of $A$, has nonempty intersection with $l$.

Let us take any $p-d$ convex sets $S_{1}, S_{2}, \ldots, S_{p-d}$ in $\mathcal{F}$ different from all the $K_{i}$ and consider the $p$ convex sets $S_{1}, S_{2}, \ldots, S_{p-d}, K_{1}, K_{2}, \ldots, K_{d}$. Every intersecting $q$-tuple cannot contain all the $K_{i}$. However, every $K_{i}$ must be in at least $d-1$ of the intersecting $q$-tuples. So every $K_{i}$ is missing in exactly one of the $q$-tuples. Let $p_{i}$ be the point of intersection of the $q$-tuple missing $K_{i}$. Then $p_{i} \in \bigcap_{j \neq i} K_{j}$. With this we have that the convex hull of the $p_{i}$ intersects $l$.

There are at least $q d$ pairs $\left(R, p_{j}\right)$ such that $R$ is one of $S_{1}, S_{2}, \ldots, S_{p-d}$, $K_{1}, K_{2}, \ldots, K_{d}$ and $p_{j} \in R$. Any $R$ cannot be in more that $d$ of those pairs, so there are at least $q d-p(d-1)$ of the convex sets in $d$ of the pairs. This cannot be one of the $K_{i}$, so there are at least $q d-p(d-1)$ of the $S_{i}$ in $d$ of the pairs. Those convex sets contain each of the $p_{i}$ and thus have a common point of intersection with $l$.

This tells us that the family $\mathcal{F} \backslash A$ has the $(p-d, q d-p(d-1))$ property in $l$. Since $q d-p(d-1) \geq 2$, they can be pierced with at most $(p-d)-[q d-$ $p(d-1)]+1=d(p-q-1)+1$ points.

Note that in the proof of the lemma it is implicit that all the $K_{i}$ are not empty.

Theorem 1 Let $\mathcal{F}$ be a finite family of convex sets $\mathcal{F}$ in $\mathbb{R}^{d}$, and p, q positive integers such that $q d>p(d-1)+1$. If $\mathcal{F}$ has at least $p$ convex sets and the $(d-1)$-balanced $(p, q)_{d}$ property, then

$$
\pi(\mathcal{F}) \leq d(p-q-1)+2
$$

Proof The theorem holds for $d=1$, so we may take $d \geq 2$. Since $\mathcal{F}$ has at least $p$ convex sets, not all its $d$-tuples have empty intersections. We know that there is a direction $v$ such that every intersecting $d$-tuple has its $v$-directional minimum in a single point. Let $A=\left\{K_{1}, K_{2}, \ldots, K_{d}\right\}$ be the intersecting $d$-tuple with maximal $v$ directional minimum $p_{0}$. We know that every set in $\mathcal{F}$ that has nonempty intersection with $K_{1}, K_{2}, \ldots, K_{d}$ contains $p_{0}$. Let $H=\left\{x \mid x \cdot v<x \cdot p_{0}\right\}$ and let $A^{\prime}=\left\{K_{i} \cap H \mid\right.$ $1 \leq i \leq d\}$. Let $B$ be the set of convex sets in $\mathcal{F}$ that do not contain $p_{0}$. We define

$$
\mathcal{F}^{\prime}=\{K \cap H \mid K \in B\} \cup A^{\prime} .
$$

Given the maximality of $p_{0}$, since the $v$-directional minimum of an intersection of $d+1$ convex sets is the $v$-directional minimum of some $d$ of those convex sets, every 
$d+1$ convex sets in $\mathcal{F}^{\prime}$ have nonempty intersection if and only if they had nonempty intersection in $\mathcal{F}$. This means that $\mathcal{F}^{\prime}$ also has a $(d-1)$-balanced $(p, q)_{d}$ property and $A^{\prime} \subset \mathcal{F}^{\prime}$ is a nonintersecting $d$-tuple.

We have two cases:

Case 1: There are at least $p$ convex sets in $\mathcal{F}^{\prime}$. Applying Lemma 3, we know that $\mathcal{F}^{\prime} \backslash A^{\prime}$ can be pierced with at most $d(p-q-1)+1$ points. Adding $p_{0}$, we have that $\mathcal{F}$ can be pierced with at most $d(p-q-1)+2$ points.

Case 2: There are less than $p$ convex sets in $\mathcal{F}^{\prime}$. Let $C$ be a set of $p-\left|\mathcal{F}^{\prime}\right|$ sets in $\mathcal{F}$. If we take $A \cup B \cup C$ we must have at least one intersecting $q$-tuple. If this $q$-tuple contains every one of the $K_{i}$, it cannot have any convex set of $B$. Then, we can pierce the sets of $B$ with at most $p-q$ points and the rest of the convex sets with $p_{0}$. If there is at least one of the $K_{i}$ that the $q$-tuple does not contain, then the $q$-tuple is missing at most $p-q-1$ sets of $B$. If we also use a point in the intersection of the $q$-tuple and $p_{0}$, we also get $p-q+1$ points. Since $d(p-q-1)+2 \geq p-q+1$, we are done.

Corollary 1 Let $p>d+1$ be positive integers. If $\mathcal{F}$ is a family of at least $p$ convex sets in $\mathbb{R}^{d}$ with the $(p, p-1)_{d}$ property, then $\mathcal{F}$ can be pierced with at most 2 points.

Proof All $p$ convex sets have $d$ intersecting ( $p-1)$-tuples. However, every $(p-1)$ tuple is missing exactly one convex set, so every convex set is in at least $d-1$ of the intersecting $(p-1)$-tuples. Since $p>d+1$, we have that $d(p-1)>p(d-1)+1$. Since the conditions of Theorem 1 are met, $\mathcal{F}$ can be pierced with at most 2 points.

\section{Unbalanced Families}

Given Corollary 1, it is natural to start looking for bounds for piercing numbers of families that do not have any balance condition. Of course, the number of intersecting $q$-tuples we need is larger in these cases.

Theorem 2 Let $\mathcal{F}$ be a finite family of convex sets in $\mathbb{R}^{d}$. If there are $s \leq d$ nonintersecting convex sets in $\mathcal{F}$ and $\mathcal{F}$ has $a(p, p-2)_{r}$ property with $p>s+2$ and $r>\left(\begin{array}{c}p \\ 2\end{array}\right)-\left(\begin{array}{c}p-s \\ p-s-2\end{array}\right)-(p-d)$, then $\mathcal{F}$ can be pierced using at most 4 points.

Proof First we will prove that all $s-1$ convex sets in $\mathcal{F}$ are intersecting. If we can find $s-1$ nonintersecting convex sets, let us complete them with $p-s+1$ other convex sets. We know that out of those $p$ sets, every $(p-2)$-tuple that contains the $s-1$ nonintersecting convex sets is nonintersecting. Thus, there are at least $\left(\begin{array}{c}p-s+1 \\ p-s-1\end{array}\right)$ of the $(p-2)$-tuples which are not intersecting. Note that $\left(\begin{array}{c}p \\ 2\end{array}\right)-\left(\begin{array}{c}p-s \\ p-s-2\end{array}\right)-(p-d) \geq$ $\left(\begin{array}{l}p \\ 2\end{array}\right)-\left(\begin{array}{c}p-s+1 \\ p-s-1\end{array}\right)$ is equivalent to $p-s=\left(\begin{array}{c}p-s \\ p-s-1\end{array}\right)=\left(\begin{array}{c}p-s+1 \\ p-s-1\end{array}\right)-\left(\begin{array}{c}p-s \\ p-s-2\end{array}\right) \geq p-d$. Since $s \leq d$, this is true. So, we have at least $r$ intersecting $(p-2)$-tuples and therefore, we cannot have $s-1$ nonintersecting sets.

By Lemma 1 , since we have $s$ nonintersecting sets $K_{1}, K_{2}, \ldots, K_{s}$, there is an affine subspace $\Pi$ of dimension $d+1-s$ such that if any convex has nonempty 
intersection with every $s-1$ of the $s$ nonintersecting convex sets, then it has a nonempty intersection with $\Pi$.

Let $B=\left\{H_{1}, H_{2}, \ldots, H_{p-s}\right\}$ be any other $p-s$ convex sets in $\mathcal{F}$, and $A=$ $\left\{K_{1}, K_{2}, \ldots, K_{s}\right\}$. If we take the $p$-tuple $A \cup B$, we know that every $(p-2)$-tuple containing $A$ is nonintersecting, which gives us at least $\left(\begin{array}{c}p-s \\ p-s-2\end{array}\right)$ nonintersecting $(p-2)$-tuples. Of all the other $(p-2)$-tuples, we know that at most $p-d-1$ are nonintersecting. Thus, at most $p-d-1$ of the $(p-s-1)$-tuples of $B$ do not make a nonempty intersecting $p-2$ tuple with every $(s-1)$-tuple of $A$. Since there are $p-s$ of those $(p-s-1)$-tuples, then at least $d+1-s$ of those $(p-s-1)$-tuples form a nonempty $(p-2)$-tuple with every $(s-1)$-tuple of $A$. This gives a point of their intersection in $\Pi$. Thus, $\mathcal{F} \backslash A$ has a $(p-s, p-s-1)_{d+1-s}$ property in $\Pi$. By Corollary $1, \mathcal{F} \backslash A$ can be pierced with at most 2 points.

Since all $s-1$ sets in $\mathcal{F}$ are intersecting, $A$ can be pierced with at most 2 points as well.

The previous result can be extended if $s=d$.

Lemma 4 Let $r, p, q, d$ be positive integers such that $p>q>d$ and let $\mathcal{F}$ be a family of at least $p$ convex sets in $\mathbb{R}^{d}$ with the $(p, q)_{r}$ property. If $r>\left(\begin{array}{l}p \\ q\end{array}\right)-\left(\begin{array}{l}p+1-d \\ q+1-d\end{array}\right)$ and there is a nonintersecting $d$-tuple $A \subset \mathcal{F}$, then

$$
\pi(\mathcal{F} \backslash A) \leq p-q .
$$

Proof The proof of this lemma is similar to the proof of Lemma 3; however, instead of using the balance condition to obtain some kind of $(p, q)$ property on a line, we use that $r$ is large enough for that to happen.

Define $K_{1}, K_{2}, \ldots, K_{d}$ and $l$ as in the proof of Lemma 3. Let $B=\left\{H_{1}, \ldots, H_{p-d}\right\}$ be any other $p-d$ convex sets in $\mathcal{F}$. We know that every $q$-tuple in $A \cup B$ containing $A$ is nonintersecting. This gives us $\left(\begin{array}{c}p-d \\ p-q\end{array}\right)$ nonintersecting $q$-tuples. Thus, we have at most $\left(\begin{array}{l}p \\ q\end{array}\right)-\left(\begin{array}{l}p-d \\ p-q\end{array}\right)-r q$-tuples which do not contain $A$ and are nonintersecting.

Consider all the pairs $(R, S)$, where $R$ is a $(q-d+1)$-tuple from $B, S$ is a $(d-1)$ tuple from $A$, and $R \cup S$ is an intersecting $q$-tuple. Since there are $\left(\begin{array}{c}p-d \\ q+1-d\end{array}\right)$ possible choices for $R$, then there are at least $\left(\begin{array}{c}p-d \\ q+1-d\end{array}\right)-\left[\left(\begin{array}{l}p \\ q\end{array}\right)-\left(\begin{array}{c}p-d \\ p-q\end{array}\right)-r\right]=r-\left(\left(\begin{array}{l}p \\ q\end{array}\right)-\right.$ $\left.\left[\left(\begin{array}{c}p-d \\ q-d\end{array}\right)+\left(\begin{array}{c}p-d \\ q+1-d\end{array}\right)\right]\right)>0$ of the $R$ 's which make one of these pairs with every possible choice for $S$. This $(q-d+1)$-tuple has a point in common with $l$. Thus, $\mathcal{F} \backslash A$ has the $(p-d, q+1-d)$ property in $l$. Since $q+1-d \geq 2$, they can be pierced with at most $p-q$ points.

Using this lemma, we can also prove a general theorem. As in Lemma 3, the nonemptiness of the $K_{i}$ is implicit in the proof.

Theorem 3 Let $r, p, q, d$ be positive integers such that $p>q>d$ and let $\mathcal{F}$ be a family of at least $p$ convex sets in $\mathbb{R}^{d}$ with the $(p, q)$ r property. If $r>\left(\begin{array}{l}p \\ q\end{array}\right)-\left(\begin{array}{l}p+1-d \\ q+1-d\end{array}\right)$, then

$$
\pi(\mathcal{F}) \leq p-q+1
$$


Proof The theorem holds for $d=1$, so we take $d \geq 2$. Define $v, K_{1}, K_{2}, \ldots, K_{d}, p_{0}$, $H, A, B, A^{\prime}$, and $\mathcal{F}^{\prime}$ as in the proof of Theorem 1. Again, we have two cases.

Case 1: There are at least $p$ convex sets in $\mathcal{F}^{\prime}$. Applying Lemma 4 to $\mathcal{F}^{\prime}$, we obtain that $\mathcal{F}^{\prime} \backslash A^{\prime}$ can be pierced with at most $p-q$ points. Adding $p_{0}$, we obtain that $\mathcal{F}$ can be pierced with at most $p-q+1$ points.

Case 2: There are less than $p$ convex sets in $\mathcal{F}^{\prime}$. With the same arguments of the second case of the proof of Theorem 1 , we can pierce $\mathcal{F}$ using at most $p-q+1$ points.

If $d=1$, our theorem gives the piercing number, for a family of intervals in the line, with the $(p, q)$ property. If $p=q+1$, this theorem gives the same corollary as Theorem 1. If $d=2$, we have the following corollary.

Corollary Let $p, n$ be positive integers such that $p \geq n+2$ and $n \geq 2$. Let $\mathcal{F}$ be $a$ family of at least $p-1$ convex sets in $\mathbb{R}^{2}$ with the $(p-1, p-x)^{\frac{n}{p}}$ property. Then

$$
\pi(\mathcal{F}) \leq n
$$

For example, if for every $2 n-1$ convex sets of $\mathcal{F}$ more than half of its $n$-tuples are intersecting, then $\mathcal{F}$ can by pierced with $n$ points.

Finally, we will show a lower bound for $r$ in order for the $(p, q)_{r}$ property to imply that the piercing number of the family is at most $k$.

Claim 1 Let $p, q, r, k, d$ be positive integers, $p>q>d, p \geq k$. If every family of

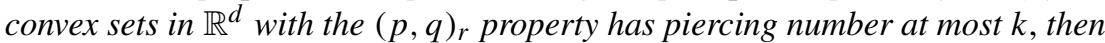

$$
r>\left(\begin{array}{c}
p-k-1 \\
q
\end{array}\right)+(k+1)\left(\begin{array}{c}
p-k-1 \\
q-1
\end{array}\right) .
$$

Proof It suffices to show a family $\mathcal{F}$ of convex sets with the $(p, q)_{r}$ property where $r=\left(\begin{array}{c}p-k-1 \\ q\end{array}\right)+(k+1)\left(\begin{array}{c}p-k-1 \\ q-1\end{array}\right)$ and piercing number at least $k+1$. Let $\mathcal{F}$ be a family of $n$ convex sets, $k+1$ of which are points and where the rest are convex sets that contain all these points. A simple counting argument shows that the family has the $(p, q)_{r}$ property; however, it cannot be pierced with less than $k+1$ points.

\section{References}

1. Alon, N., Kleitman, D.J.: Piercing convex sets and the Hadwiger-Debrunner $(p, q)$-problem. Adv. Math. 96, 103-112 (1992)

2. Bracho, J., Montejano, L.: Helly type theorems on the homology of the space of transversals. J. Discrete Comput. Geom. 27(3), 387-393 (2002)

3. Hadwiger, H., Debrunner, H.: Über eine Variante zum Hellyschen Satz. Arch. Math. 8, 309-313 (1957) (German)

4. Kleitman, D.J., Gyárfás, A., Tóth, G.: Convex sets in the plane with three of every four meeting. Combinatorica 21(2), 221-232 (2001) 